\title{
Activity recognition based on accelerometer sensor using combinational classifiers
}

\begin{abstract}
In recent years, people nowadays easily to contact each other by using smartphone. Most of the smartphone now embedded with inertial sensors such accelerometer, gyroscope, magnetic sensors, GPS and vision sensors. Furthermore, various researchers now dealing with this kind of sensors to recognize human activities incorporate with machine learning algorithm not only in the field of medical diagnosis, forecasting, security and for better live being as well. Activity recognition using various smartphone sensors can be considered as a one of the crucial tasks that needs to be studied. In this paper, we proposed various combination classifiers models consists of J48, Multi-layer Perceptron and Logistic Regression to capture the smoothest activity with higher frequency of the result using vote algorithm. The aim of this study is to evaluate the performance of recognition the six activities using ensemble approach. Publicly accelerometer dataset obtained from Wireless Sensor Data Mining (WISDM) lab has been used in this study. The result of classification was validated using 10fold cross validation algorithm in order to make sure all the experiments perform well.
\end{abstract}

Keyword: Classification; Accelerometer; Activity; Sensors 